\title{
Comparison of Subgingival Microbiota in Fluorosed and Non-fluorosed Chronic Periodontitis Patients
}

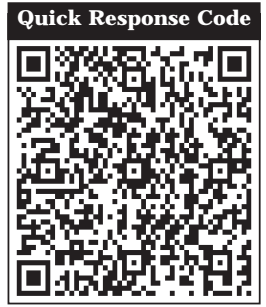

doi: $10.5866 / 2015.7 .10026$

1Post Graduate

${ }^{3}$ Professor

${ }^{4}$ Reader

${ }^{5}$ Professor and HOD

Department of Periodontics

Kamineni Institute of Dental Sciences, Narketpally.

${ }^{2}$ Private practitioner Anantapur.

\section{Article Info:}

Received: J anuary 8, 2015

Review Completed: February 9, 2015

Accepted: March 8, 2015

Available Online: April, 2015 (www.nacd.in)

(c) NAD, 2015 - All rights reserved

\section{Email for correspondence:}

abhinav_gupta22@yahoo.com

\author{
Abhinav Atchuta ${ }^{1}$, Chandra Sekhara Reddy S², \\ Satyanarayana $\mathrm{D}^{3}$, J agadish Reddy $\mathrm{G}^{4}$, Rajababu $\mathrm{P}^{5}$
}

\begin{abstract}
:
Background: Periodontal disease comprises a group of inflammatory conditions of the supporting tissues of the teeth that are caused by bacteria. Only few species of subgingival microbiota have been identified as etiol ogic pathogens. The roots of teeth with mottled enamel are irregular and rough which favor plaque accumulation. The present study aimed at assessing the microbial composition of subgingival plaque in Fluorosed and Non Fluorosed Chronic Periodontitis subjects using PCR. Materials and Method: 40 chronic periodontitis patients were selected and divided into fluorosed and nonfluorosed groups. The Oral hygiene status, fluorosis status and periodontal status were recorded and compared. After recording the clinical parameters, at least 3 sites of 5-7 mm of pocket depth were selected for subgingival pooled plaque sample collection. Samples were subjected to culture and PCR analysis. Results: The PCR results for A. actinomycetemcomitans, P. gingivalis, $T$. forsythia and $T$. denticola organisms when compared between the groups showed nostatistically significant difference. But for $\mathrm{T}$. forsythia and $\mathrm{T}$. denticolastatistically significant difference was observed. Conclusion: The patients in fluorosed group showed more positive findings for periodontitis when compared to non-fluorosed group. Severity of periodontal disease was directly proportionate with the degree of fluorosis when compared within the fluorosed group. Qualitative comparison of subgingival microbiota showed no significant differences between the fluorosed and non fluorosed group. The specific detection rates of $\mathrm{T}$. forsythia and $\mathrm{T}$. denticola is more in the fluorosed group than the non fluorosed group.
\end{abstract}

Key words: Chronic Periodontitis, Dental Plaque, Polymerase Chain Reaction, Dental Fluorosis, Microbiota

\section{INTRODUCTION:}

Periodontal disease comprises a group of inflammatory conditions of the supporting tissues of the teeth that are caused by bacteria. If left untreated, the teeth lose their ligamentous support to the alveolar bone, become mobile, and are eventually lost. Numerous risk factors have been described which include a genetic background, systemic conditions, socioeconomic status, environmental factors, smoking habits, past periodontal breakdown, immunologic and microbial parameters. $^{1-6}$ 
Although the subgingival microenvironment in the periodontal pocket is characterized by a wide diversity, with over 600 species having been isolated from different individuals and as many as 40 from a single site, but only a few species have been associated with disease. ${ }^{7}$ A limited number have demonstrated a clear etiological role and these have been identified as periodontal pathogens. ${ }^{8}$ E vidence for etiology is based on fulfillment of several criteria described by Socransky(1970). ${ }^{9}$ Using these criteria, it has been demonstrated that microbes $A$. acomitans, P. gingivalis, T. forsythia, T. denticola play a clear etiological role in periodontal disease.

From microbiological viewpoint, tooth provides a hard, non shedding surface that allows the development of extensive bacterial deposits. The accumulation and metabolism of bacteria on these hard surfaces are the primary cause for gingivitis, periodontitis, often bad breath and caries. ${ }^{10}$

Although surface free energy and surface roughness are two factors influencing plaque growth, the latter predominates. ${ }^{11}$ The roots of teeth with mottled enamel, are irregular, rough and revealed heavy deposits of calcified marks in the form of excessive amounts of fluoride or osteocementum at the apical region of the teeth.12 Fluoride is used therapeutically to aid in remineralization of tooth structures, prevention of demineralization of tooth structures and inhibition of the growth of many plaque microorganisms. ${ }^{13}$

Microbial identification has been based on phenotypic and biochemical criteria, including microscopy, bi ochemical reactivity, growth conditions, dye and immunoflorescence staining, bacterial end-product analysis, cell membrane composition and antibiotic sensitivity. Most predominant bacterial species in the oral cavity have been identified using culture-independent molecular method based on nucleic acid sequence analysis. ${ }^{14}$

The study aimed at assessing the microbial composition of subgingival plaque in fluorosed and non fluorosed chronic periodontitis subjects using PCR and to compare the subgingival microbiota of fluorosed chronic periodontitis subjects with Non Fluorosed Chronic periodontitis subjects.

\section{Materials and methods:}

Periodontitis (AAP, 1999) patients were divided into fluorosed and non-fluorosed group based on modified Dean's dental fluorosis index. Depending on the severity of fluorosis, assessed using Modified Dean's fluorosis Index and the patients were randomly assigned. ${ }^{15,} 16$
Patients included in the study were within the age group of 20-50 years who were diagnosed with chronic periodontitis based on periodontal disease index with periodontal pockets ranging from $5-7$ $\mathrm{mm}$ and had at least 8 teeth and 20 sites involved. Subjects with dental fluorosis were determined by Modified Dean's fluorosis Index.

Subjects aged below 20 years and above 50 years with any known systemic diseases, who had received any antibiotic or periodontal therapy in the last 6 months, with aggressive periodontitis, with intrinsic staining due to other causes, with periodontal pockets $<5 \mathrm{~mm}$ and $>7 \mathrm{~mm}$, who were pregnant or lactating and smokers were excluded from the study.

A total number of 40 chronic periodontitis patients of which 20 patients are affected by dental fluorosis (Figurel) and 20 free from dental fluorosis were selected for the study (Figure 2). The oral hygiene status, fluorosis status and periodontal status were recorded and compared between the patients of both the groups using the following Indices

1. Oral Hygiene index (Greene and Vermillion 1964) $)^{17}$

2. Periodontal Disease Index (PDI) (Ramfjord, 1959) ${ }^{\mathbf{1 8}}$ (Figure 3)

3. Modified Dean's Fluorosis I ndex (Dean, 1942) ${ }^{16}$

The clinical parameters compared between the patients of both the groups were Probing Pocket Depth and Clinical Attachment Level.

\section{Plaque sample collection:}

After recording the clinical parameters, at least 3 sites of 5-7 mm of pocket depth were selected for subgingival pooled plaque sample collection. The sample site was isolated with sterile cotton rolls and the supragingival plaque was removed using cotton rolls and air dried. A sterile curette was introduced to the base of the pocket and plaque was removed (Figure 4). The curette with the collected plaque was dispensed in a test tube containing normal saline, which was immediately centrifuged and the supernatant was discarded and $0.5 \mathrm{ml}$ of the deposit is transferred to a vial containing transport media viz. TE buffer (10mM Tris-HCL, ImM EDTA pH 8). The vial was closed, labelled and sent to the microbiological laboratory within 48 hours of collection.

\section{Microbial analysis:}

The pooled plaque samples were subjected to Culture and Multiplex Polymerase Chain Reaction (PCR) analysis to identify Aggregatibacter 
actinomycetemcomitans, Porphyromonasgingivalis, Tannerella forsythia and Treponemadenticola (Figure 5)

\section{Processing of samples:}

The samples were stored at $-20^{\circ} \mathrm{C}$ in the laboratory and DNA extraction and multiplex PCR were performed.

\section{Statistical Analysis:}

Mann-Whitney U-test is used to test whether two independent groups have been drawn from the same population. Kruskal Wallis ANOVA test by ranks is used find out the significance differences between more than two groups or the comparable test for ordinal data.

\section{Results}

The mean age range of fluorosed patients included in the study was $46.35 \pm 3.69$, and nonfluorosed patients had a mean age range of 43.5 \pm 6.03 . The periodontal disease Index scores were compared between the fluorosed and non-fluorosed groups and the results showed no statistically significant differenceand when compared within the fluorosed group between the mild, moderate and severe subgroups (Table I).

The PCR results for $A$. actinomycetemcomitans, $P$. gingivalis, $T$. forsythia and $T$. denticola organisms were compared between fluorosed and non fluorosed groups showed statistically no significant difference among them between PCR results (Table II). The comparison of PCR results for $A$. actinomycetemcomitans and $P$. gingivalis between mild, moderate and severe subgroups showed no significant difference. But for $T$. forsythia and $T$. denticola statistically significant difference was observed (Table III).

\section{Discussion:}

Periodontal diseases are multifactorial infections elicited by a complex of bacterial species that interact with host tissues and cells which lead to the destruction of the periodontal structures, which include alveolar bone, and periodontal ligament. ${ }^{19}$ The trigger for the initiation of disease is the presence of complex microbial biofilms that colonize the sulcular regions between the tooth surface and the gingival margin through specific adherence interactions and accumulation due to architectural changes in the sulcus (i.e. attachment loss and pocket formation). ${ }^{20}$

The development of periodontal disease has been thought to be associated with several restricted members of oral anaerobic species such as A. actinomycetemcomitans, $P$. gingivalis, $T$. denticola and $T$. forsythia which are frequently found in the dental plaque. ${ }^{21}$ With the acceptance of specific plaque hypothesis, and increased knowledge on the role of anaerobic microbial species in periodontal disease progression, emphasis is now being laid on eliminating these organisms.

Periodontal disease severity distribution, progression depends on various microbial, host, environmental and local factors. ${ }^{22}$ Among the effect of various environmental etiological factors, the influence of fluoride on the periodontal health is still controversial. Although studies have been conducted on the effect of elevated fluoride in drinking water on gingivitis and periodontitis, the results have been inconsistent, higher level of gingival inflammation has been observed in high fluoride areas than nonfluoride areas. ${ }^{23,24}$

Although fluoride decreases the caries incidence, the effects of fluoride on inflammatory periodontal disease is obscure. ${ }^{25} \mathrm{Nalgonda}$ district is a known endemic fluoride area in the state of Andhrapradesh. ${ }^{26}$ During delivery of routine periodontal care in the department of Periodontics, Kamineni Institute of Dental Sciences, Narketpally, $\mathrm{Nalgonda}$ district, it has been observed that a majority of patients are affected with fluorosis. This has prompted to take up the present study to assess any difference in the microbiota in fluorosed and non fluorosed patients in chronic periodontitis patients. Hence the present study was undertaken to compare microorganisms related to periodontitis viz. bacteria such as $A$. actinomycetemcomitans, P. gingivalis, $T$. forsythia and $T$. denticola, in vivo.

In the present study when the periodontal status was compared with varying severity of fluorosis (mild, moderate and severe subgroups), statistically significant difference was observed. As the severity of fluorosis increased, gradual increase in the PDI scores were found. This may be because of higher percentage of hyper mineralized surface, resorption bays/cavitations, partial/initial mineralization of connective tissue fibers, insertion area for fibers; gl obular mineralization debris than the non-Fluorosed group. ${ }^{27}$

Within the fluorosis group, the presence of the four organisms were analyzed and compared between mild, moderate and severe fluorosis subgroups using Kruskal Wallis ANOVA TEST and further comparison between the subgroups was done using Mann Whitney $U$ test. The comparison revealed statistically insignificant difference in the 
Table I: Distribution of study subjects based on gender according to fluorosed and non-fluorosed groups with mean standard deviation and PDI scores

\begin{tabular}{lcccc} 
& \multicolumn{2}{c}{ Gender $(\mathbf{n} \%)$} & Mean & \\
Male & Female & & PDI scores \\
\hline Fluorosed & $50(10)$ & $50(10)$ & $46.35 \pm 3.69$ & $5.29 \pm 0.18$ \\
\hline Non-Fluorosed & $45(9)$ & $55(11)$ & $43.5 \pm 6.03$ & $5.29 \pm 0.26$ \\
\hline
\end{tabular}

Table II: Comparison of fluorosed and non-fluorosed groups with respect to PCR results and PCR counts (cells/ml of Plaque)

\begin{tabular}{llccccc} 
Variable & Group & \multicolumn{2}{c}{ PCR } & & \multicolumn{2}{c}{ PCR COUNT } \\
\cline { 3 - 3 } & & Mean \pm SD & P-value & & Mean \pm SD & P-value \\
A.a & Fluorosed & $3000 \pm 4439.22$ & & & $1.6316 \pm 0.4956$ & \\
\hline & Non-Fluorosed & $5440 \pm 6191.20$ & 0.2270 & $1.5500 \pm 0.5104$ & 0.6632 \\
\hline P.g & Fluorosed & $7063.16 \pm 4673.83$ & & $1.1579 \pm 0.3746$ & \\
\hline & Non-Fluorosed & $5620 \pm 3282.43$ & 0.2794 & $1.1500 \pm 0.3663$ & 0.9664 \\
\hline T.f & Fluorosed & $2840 \pm 3688.16$ & & $1.4737 \pm 0.5130$ & \\
\hline & Non-Fluorosed & $5168.42 \pm 6443.35$ & 0.3837 & & $1.5500 \pm 0.5104$ & 0.6837 \\
\hline T.d & Fluorosed & $960 \pm 1998.53$ & & $1.6842 \pm 0.4776$ & \\
\hline & Non-Fluorosed & $3288.89 \pm 5331.03$ & 0.2993 & $1.7500 \pm 0.4443$ & 0.7254 \\
\hline
\end{tabular}

$* \mathrm{p}<0.05$

Table III: Comparison of fluoride levels (mild, moderate and severe) with respect to A. actinomycetemcomitans, P. gingivalis, T. forsythia, T. denticola (PCR) and PCR counts (cells/ml of plaque) in fluorosed groups

\begin{tabular}{llcccc} 
Variable & Group & \multicolumn{2}{c}{ PCR } & \multicolumn{2}{c}{ PCR COUNT } \\
& & Mean \pm SD & P-value & Mean \pm SD & P-value \\
A.a & Mild & $0.50 \pm 0.53$ & 0.0778 & $3600 \pm 4097.70$ & 0.0694 \\
\hline & Moderate & 0.00 & & 0.00 & \\
\hline & Severe & $0.67 \pm 0.58$ & & $7000 \pm 7000$ & \\
\hline P.g & Mild & $0.80 \pm 0.42$ & 0.7180 & $6840 \pm 4873.33$ & 0.9864 \\
\hline & Moderate & $0.83 \pm 0.41$ & & $7300 \pm 5093.92$ & \\
\hline & Severe & $1 \pm 0$ & & $7333.33 \pm 4932.88$ & \\
\hline T.f & Mild & $0.10 \pm 0.32$ & $0.0007 *$ & $560 \pm 1770.88$ & $0.0007^{*}$ \\
\hline & Moderate & $1.00 \pm 0.00$ & & $10166.7 \pm 3793.5$ & \\
\hline & Severe & $1.00 \pm 0.00$ & & $10533.3 \pm 9962.60$ & \\
\hline T.d & Mild & $0.00 \pm 0.00$ & $0.0033^{*}$ & $0.0 \pm 0.00$ & $0.0045^{*}$ \\
\hline & Moderate & $0.50 \pm 0.55$ & \multicolumn{3}{c}{$4533.3 \pm 5834.61$} \\
\hline
\end{tabular}

$* p<0.05$ 


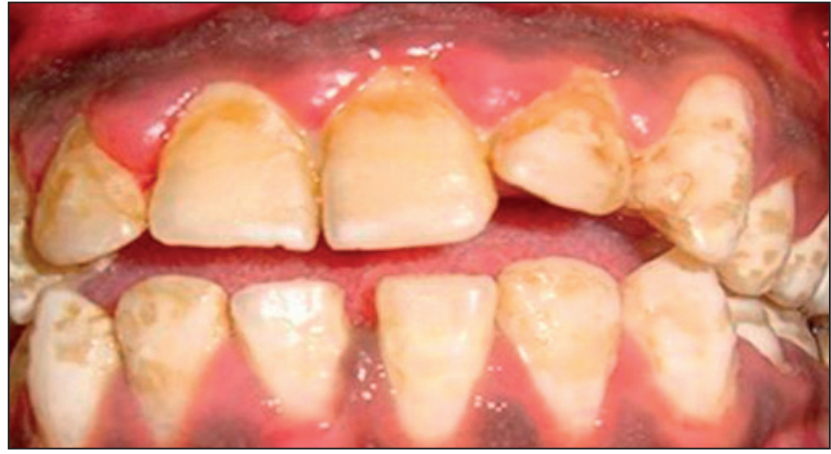

Figure 1: Dental fluorosis

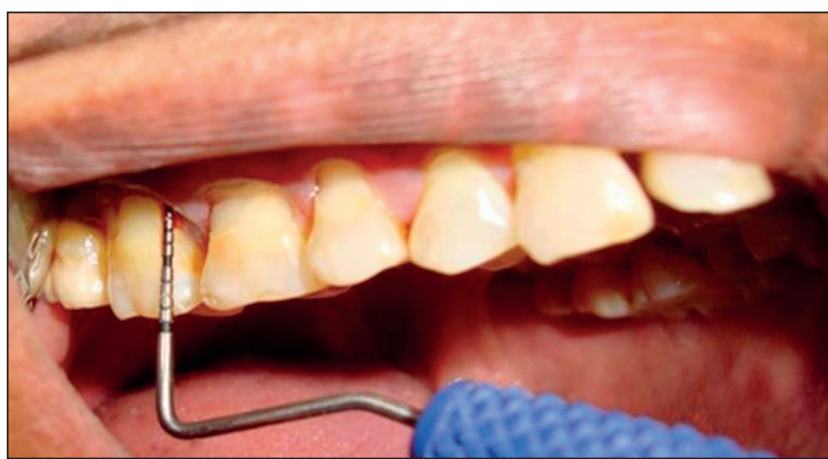

Figure 3: Recording Periodontal Disease Index (PDI)

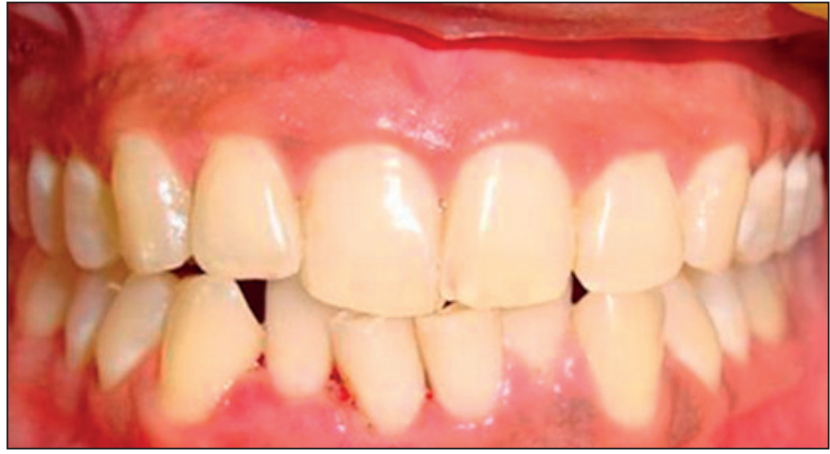

Figure 2: Non-fluorosed teeth

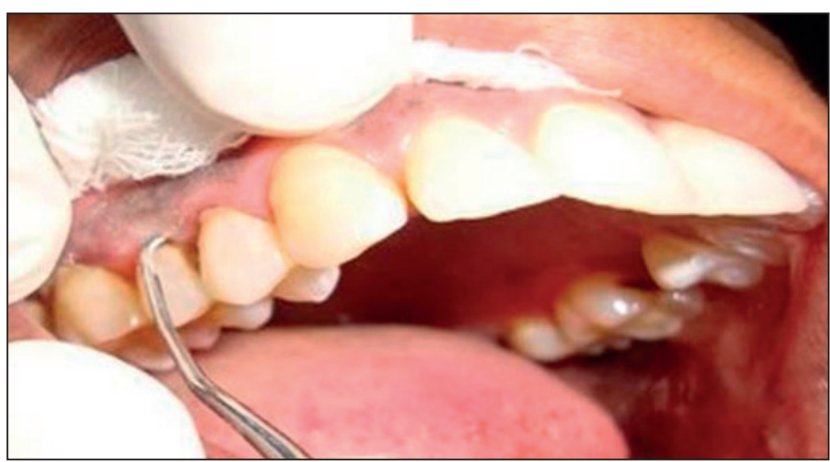

Figure 4: Plaque sample collection

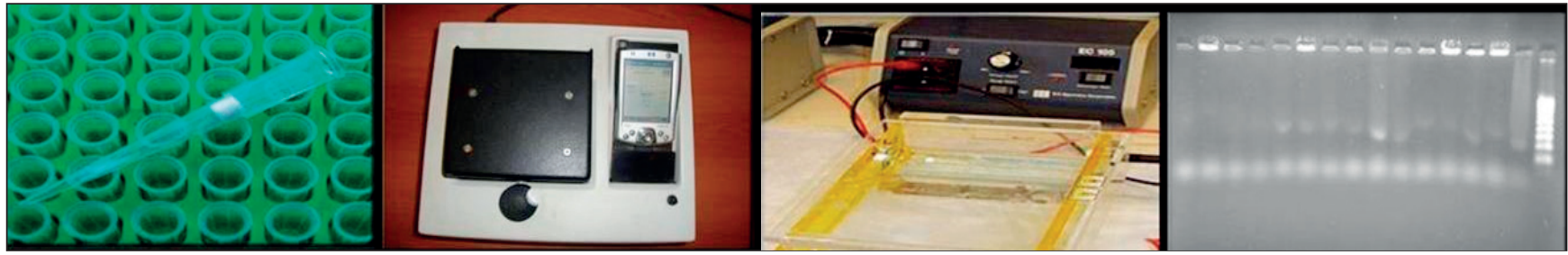

Figure 5: Armamentarium for PCR analysis

detection rates of $A$. actinomycetemcomitans and $P$. gingivalis.

However the detection rates of $\mathrm{T}$. forsythia were higher in moderate and severe fluorosis subgroups and these differences when compared with Kruskal Wallis ANOVA TEST were statistically significant. Similar significant differences were verified by further comparison using Mann Whitney $U$ test. Comparison of detection rates of $\mathrm{T}$. denticola was done using Kruskal Wallis ANOVA TEST and higher detection rates were observed in severe fluorosis when compared to moderate and mild subgroups. These differences were statistically significant. Further comparison using Mann Whitney $U$ test revealed these differences to be statistically significant between mild and severe subgroups.
The comparison revealed statistically insignificant difference in the number of $A$. actinomycetemcomitans and $P$. gingivalis in the plaque samples. Highest quantities of $\mathrm{T}$. forsythia and $\mathrm{T}$. denticola were detected in severe fluorosis group and the difference was statistically significant. However the quantity of $\mathrm{T}$. denticola when compared between mild, moderate and severe subgroups revealed statistically significant difference between mild and severe fluorosis subgroups.

As the microbial evaluation within the subgroups of fluorosis patients were not done earlier, the results of the study could not be compared. The interesting finding that increased PDI scores and higher detection rates and quantities of periodontal pathogens especially $T$. forsythia and $T$. denticola was seen in moderate and severe fluorosis patients which further necessitates us to understand the 
exact influence of fluorosis on the subgingival pocket environment and the microbial colonization. As the present study forms an initial step in revealing the influence of fluorosis on subgingival microbiota, to reconfirm and validate the results further studies with larger sample size and evaluation using longitudinal study designs for extended periods of time are needed.

\section{Conclusion:}

All the selected patients belonging to both groups were age matched and belonged to rural population and the patients belonging to both the groups were gender matched. The patients in fluorosed group showed more positive findings for Periodontitis when compared to non-fluorosed group. Periodontal disease severity was directly proportionate with the degree of fluorosis when compared within the fluorosed group among mild and moderate subgroups. The qualitative comparison of subgingival microbiota showed no significant differences between the groups in the combined detection rates of $A$. actinomycetemcomitans, P. gingivalis, and T.forsythia and $\mathrm{T}$. denticola between the fluorosed and non fluorosed group. The specific detection rates of $\mathrm{T}$. forsythia and $\mathrm{T}$. denticola is more in the fluorosed group than the non fluorosed group.

\section{References:}

1. Nibali L, Donos N, Brett PM, et al. A familial analysis of aggressive periodontitis - Clinical and genetic findings. J Periodontal res 2008; 43:627-634.

2. Weidlich P, Cimoes R, Pannuti CM, Oppermann RV. Association between periodontal diseases and systemic diseases. Braz Oral Res 2008; 22:32-43.

3. Beck J D, Koch GG, Rozier RG, Tudor GE. Prevalence and risk factors for periodontal attachment loss in a population of older community dwelling blacks and whites. J Periodontol 1990; 61:521-528.

4. Moimaz SA, Zina LG, Saliba O, Garbin CA. Smoking and periodontal disease: Clinical evidence for an association. Oral Health Prev Dent 2009; 7:369-376.

5. Rodenburg J P, Van Winkelhoff AJ, Winkel EG, Goene RJ , Abbas F, de Graff. Occurrence of Bacteroides gingivalis, Bacteroides intermedius and Actinobacillus actinomycetemcomitans in severe periodontitis in relation to age and treatment history. J ClinPeriodontol 1990; 17:392-399.

6. Grbic J T, Lamster IB, Celenti RS, Fine J B. Risk indicators for future clinical attachment loss in adult periodontitisPatient Variables. J Periodontol 1991; 62:322-329.

7. Moore W.E. \& Moore L.V. The bacteria of periodontal diseases. Periodontology 2000 1994; 5:66-77.

8. Genco CA. I solation and characterization of a minor fimbria from Porphyromonasgingivalis. I nfect. I mmune1996; 11:4788-4794.
9. Socransky S.S. Relationship of bacteria to the etiology of periodontal disease. J ournal of periodontology 1970; 67:497505.

10. Leknes KN, Lie T, Wikesjo UM, et al. Influence of tooth instrumentation roughness on subgingival microbial colonization, J periodontol 1994; 65:303-309.

11. Quirynen M, Listgarten MA. Distribution of bacterial morphotypes around natural teeth and titanium implants ad modum Branemark. Clin Oral I mplanta Res 1990; 1:813.

12. Vazirani SJ , Sing A. Endemic dental fluorosis, radiological features of dental fluorosis. J Indian DentAssoc 1968; 40:299-303.

13. Rolla G, Ogaard B, Cruz RA. Topical application of fluorides on teeth; new concepts of mechanisms of interaction. J clinPeriodontol 1993; 20:105-110.

14. Dewhrist FE, Tamer MA, Ericson RE, Lau CN, Levanos VA Boches SK, Galvin J L, Paster BJ. The diversity of periodontal spirochetes by 16S rRNA analysis. Oral Microbiol Immunol 2000; 15:196-202.

15. Armitage GC. Development of classification system for periodontal disease and conditions. Ann periodontal 1999; 4:1-6.

16. Dean H.T. The investigation of physiological effects by the epidemiological method. In moulton FR, ed.Flurone and dental health washington. American association for the advancement of sciences. J Am Dent 1942; 4:134-140.

17. Greene J C, Vermillion J R. The simplified oral hygiene index. Assoc 1964; 68:25-31.

18. Rams TE, Oler J, Listgarten MA, Slots J . Utility of Ramfjord Index teeth to assess periodontal disease progression in longitudinal studies. J Clin Periodontol 1993; 20(2):147-150

19. Kortsik C, EImer A, Tamm I. Pleural effusion due to Histoplasma capsulatum and idiopathic CD14 Iymphocytopenia. Respiration 2003; 70:118-122.

20. Smalley J W, Birss AJ , Withnall R, Silver J . Interactions of Porphyromonas gingivalis with oxyhaemoglobin and deoxyhaemoglobin. Biochem J 2002; 362:239-245.

21. Slots J . Subgingival microflora and periodontal disease. J Clin Periodontol 1979; 6:351-382.

22. Socransky SS, Haffajee AD. The bacterial etiology of destructive periodontal disease: Current concepts. J Periodontol 1992; 63:322-331.

23. Vandana KL, Pauline G, Charles M Cobb. Periodontal changes in Fluorosed and non-fluorosed teeth by scanning electronic microscope. Research report Fluoride 2007; 40(2): 128-133.

24. Anuradha KP, Chandrashekar J, Ramesh N. Prevalence of periodontal disease in endemically flourosed area of Davangere taluk India. Indian J Dent Res 2002; 13(1):1519.

25. Paine L Michael, Slots J orgen, Rick K Sandra. Fluoride use in periodontal therapy. A review of theliterature. J Am Dent Assoc 1998; 129:69-77.

26. Ramamohana RaoNV, RaoN, Surya Prakash RaoK, Schuiling RD. Fluorine distribution in waters of Nalgonda District, Andhra Pradesh, India. Environ Geol 1993; 21: 84-89.

27. J ames PMC, J ackson D, Slac GL, Lawtun FE. Gingival health and dental cleanliness in English schoolchildren. Arch Oral Biol 1960; 3:57-60. 